\title{
Application of Genetic Algorithms for the Estimation of Hydraulic Conductivity
}

https://doi.org/10.2478/sgem-2018-0013

received October 21, 2017; accepted May 28, 2018.

\begin{abstract}
In the study described here model calibration was performed employing the inverse analysis using genetic algorithms (GA). The objective of analysis is to determine value of the coefficient of hydraulic conductivity, $k$. The commonly used method for the determination of coefficient of hydraulic conductivity based on Terzaghi consolidation leads to an underestimation of the value of $k$ as the Terzaghi model does not take into account the deformation of soil skeleton. Here, an alternative methodology based on genetic algorithms is presented for the determination of the basic parameters of Biot consolidation model. It has been demonstrated that genetic algorithms are a highly effective tool enabling automatic calibration based on simple rules. The values of the coefficient of hydraulic conductivity obtained with GA are of at least one order smaller than values obtained with the Terzaghi model.
\end{abstract}

Keywords: genetic algorithms; hydraulic conductivity; Biot consolidation model.

\section{Introduction}

There is still a growing increase in interest in the use of mathematical models in geotechnical design. Most commonly, these models are developed based on finite element method (FEM).

A successful simulation requires a careful model construction. A very important part of constructing the model is the calibration of model parameters. The aim of this paper is to present a methodology of automatic soil model calibration employing inverse analysis, namely

\footnotetext{
*Corresponding author: M. Bartlewska-Urban, Wrocław University of Technology, Faculty of Geoengineering, Mining and Geology, E-mail: monika.bartlewska@pwr.wroc.pl

T. Strzelecki: Wroctaw University of Technology, Faculty of Technology And Natural Sciences
}

genetic algorithms (GA). Inverse problem requires that mathematical model is closely related to the search algorithm. Building blocks of the calibration system are briefly described in this paper.

\subsection{Parameters values.}

The objective methods of parameter calibration are usually based on inverse analysis. The general assumption here is that the estimation should lead to the maximum possible imitation of the experiment by the calibrated model. With the use of inverse methods, the obtained parameters values would, in general, have rather 'effective' than 'physical' meaning. This is because of the maximal correspondence between the experiment and the simulation result would cause, as the not modelled phenomena would also manifest in the obtained parameters values. This could be a drawback for theoretical analysis, but for practical (engineering) modelling this is actually an advantage, as it allows us to include in the results aspects that are not formally included in the model.

Inverse calibration techniques are based on some search algorithms. Typical implementation would search through the calibrated parameters space to find a set of parameters leading to maximal concurrency with experimental data. The correspondence between both must be expressed as a search criteria and the most common is the least square error between experimental data and simulation results. The typical estimation procedure is presented in Figure 1.

\subsection{Model origin}

Our experiments are based on a model of multiphase medium, assuming that the solid has hydraulically connected pores or microcracks, which allow for the flow of liquid and gas filtration. A mathematical model of the creep of a porous medium defined as a multicomponent diphase body was first introduced by Biot $[13,14]$. In a 


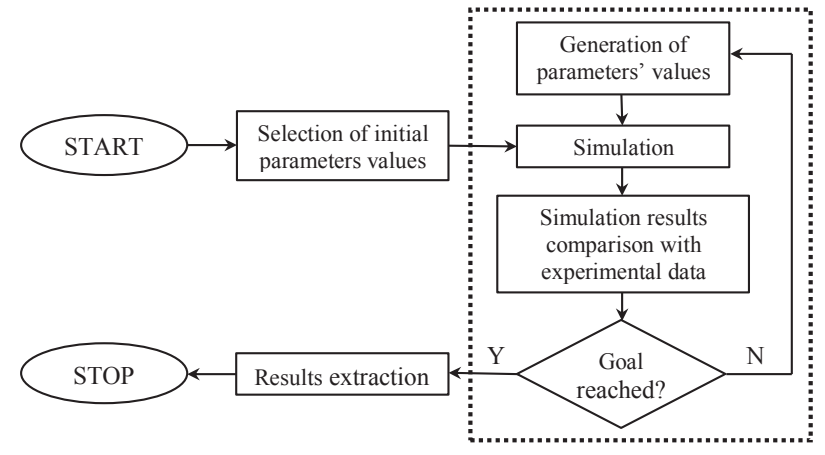

Figure 1: Inverse calibration operation diagram.

very general way the model has been analyzed on the basis of asymptotic homogenization theory for the case when the diphase medium's pores are filled with a poorly compressible liquid. For this purpose [3,5,12,21] used the methods for periodic structures while $[20,23]$ used statistical methods. For the case when the medium's pores are filled with a gas, a mathematical model based on the asymptotic homogenization method was presented by [4]. One of the fundamental assumptions of porous medium mechanics is the porous medium continuity postulate. It is a considerable simplification when this theory is applied to soils. Despite this assumption, models describing the creep of the Biot body represent well the actual processes of deformation of cohesive soils (argillaceous and clayey soils and mudclays). The processes are described also taking into account temperature changes by [26]. In [9] $\mathrm{PhD}$ thesis and in $[8,10]$ one can find consolidation equations based on the Biot model with the Kelvin-Voight rheological skeleton, showing that besides volumetric compressibility and shape elasticity, the viscosity of the soil skeleton also plays a significant role.

\section{The model and implementation of genetic algorithm}

The model calibration was performed employing inverse analysis using genetic algorithms (GA). The objective of the analysis is the estimation of filtration coefficient $k$ and its comparison with the Terzhagi methodology. In a very simplified way, GA mimic the natural evolution process in order to perform a search for the minimum of a function. In the case of calibration, the search of minim is formally optimization of a system, as we search for optimal parameter set using squared error estimator. GA have found application in many areas and are proven to be successful for the estimation of parameters.
The research was performed on clays obtained from the mine dump soil KWB Turow. An alternative way was presented to determine the coefficient of filtration than the previously commonly used method based on the Terzahgi model. That method is not complete because it does not take into account the soil skeleton deformation. The work appears to confirm the thesis that genetic algorithms are a highly effective tool for automatic analysis, based on simple rules and simultaneous calibration of all the parameters. It shows how to determine the basic parameters of the models using the example of the Biot model. The presented paper is a continuation and development of research concerning the determination of the parameters of complex rheological models [9,11].

\subsection{Mathematical model of the porous medium (Biot's model)}

The classical constitutive relations for a poroelastic body in isothermal problems were given by [14]. The effective parameters of the Biot model (Biot's constants) were described for the first time in 1957 [15] as $A, N, Q$ and $R$. The original form of the constitutive relations for a medium consisting of a porous elastic skeleton and a liquid was written as follows:

$$
\begin{aligned}
& \sigma_{i j}=2 N \varepsilon_{i j}+A \varepsilon \delta_{i j}+Q \theta \delta_{i j} \\
& \sigma-\sigma_{a}=Q \varepsilon+R \theta
\end{aligned}
$$

where $A, N, Q$ and $R$ are Biot's constants interpreted in the study of [15].

$N$ is equivalent to shear $\mathrm{r}^{-}$Jdulus $\mu$, and $A$ are is equivalent to Lame coefficient $\lambda ; R$ is the modulus of volume elasticity of the liquid; $Q$ is a coupling coefficient stemming from the mutual interaction between the solid phase and the liquid phase, $\sigma_{i j}$ is a tensor of stress in the skeleton, related to the total RVE surface, defined as a fuzzy stress tensor, $\sigma$ is the stress in the liquid, related to (similarly as the stress in the skeleton) the total surface area of the RVE cross section, also defined as fuzzy stress, $\varepsilon_{i j}$ is a skeleton deformation tensor, $\varepsilon=\varepsilon_{i i}$, a skeleton dilatation and $\theta$ is a liquid dilatation. Moreover, denoting the pressure in the liquid as $p$ and porosity as $f_{0}$, the fuzzy pore stress in the liquid is expressed by the formula $\sigma=-f_{0} p$.

Consolidation equations were derived by [14] and have the following form: 


$$
\left\{\begin{array}{l}
N \nabla^{2} u_{i}+(M+N) \varepsilon,_{i}=-\frac{H}{R} \sigma,_{i} \\
\frac{k}{f^{2}} \nabla^{2} \sigma=\frac{1}{R} \sigma-\frac{H}{R} \varepsilon
\end{array}\right.
$$

where: $\frac{k}{f^{2}}=K$, with $k$ being the Darcy's filtration coefficient and $f$ the skeleton's porosity.

In his postdoctoral thesis [27] put forward an idea of using the genetic computation technique to solve the selected geotechnical engineering problems (the stability of scarps and slopes, the interpretation of the results of triaxial compression tests, etc.). He demonstrated that the state-of-the-art numerical techniques can restore the full functionality of some solutions that have existed in geotechnics for decades, but which have not been applied in practice because of, for example, too high computing costs. Biot's model calibration based on inverse analysis, carried out by us using the genetic algorithm, has proved the belief that genetic algorithms constitute a highly effective calibration tool for the automatic (based on simple rules and simultaneous for all the parameters) determination of effective parameters, also for the more complex problems of mechanics. The results presented here can be used as the basis for further research on the application of genetic algorithms to the analysis of the effective parameters of the Biot model with the viscosity of the soil skeleton taken into account.

\subsection{Genetic algorithms}

Genetic algorithms (GAs) can be defined as a numerical technique inspired by natural processes of information processing [17]. They are classified as resistant optimization methods, or methods showing similar efficacy for a wide range of issues [19]. This flexibility derives from the fact that the optimization is performed based only on the value of the objective function. The algorithm does not have any more information of the model or of a modeled process [27]. This is a very important property of GA, as it means that for different models geometries, processes, parameters sets and experiments, they would have similar effectiveness and can be used without modification.

The methodology is, in fact, the inverse estimation. During the estimation process, the algorithm generates sets of parameters, which are used for process simulation. Based on results obtained, the estimator least-squared error is applied for valuation of the obtained solutions (Figure 1). Based on error values, more sets are generated and the process is continued until satisfying results are obtained.

GAs have proved to be very successful in many scientific and engineering areas [2,25]. A great theoretical introduction for the calibration with GAs was given by [18]. The complicated model calibration was used by $[1,22,28]$ and many others.

The terminology connected with genetic algorithms includes, beside typical optimization terms, also terms derived from genetics. This is linked with the way genetic algorithms work, which, as mentioned above, imitates natural evolutionary processes.

GAs operate on variables represented by code combinations called chromosomes. Such combinations consist of a set of characteristics (or characters) called genes. The characters belong to an (usually binary) alphabet (selected for the particular application) whose possible states are called alleles. The entire genetic information about a given element is called a genotype.

The data structures (code combinations) on which GA operates are converted into values of the each variable. Then the variables are inserted into the calibrated model and used for simulation and then evaluation by means of the objective function calculation (least square error estimator). The set of parameter values and the evaluation constitute a phenotype. A set of phenotypes processed at a given stage of optimization is called a population. Genetic operators are used when creating next populations. Due to the application of the genetic operators, a newly created generation has a different gene pool (and thus parameters values) than the preceding generation.

Figure 2 shows a block diagram illustrating how genetic algorithms work. Seven principal steps can be distinguished in the diagram [24].

The first step in the operation of GAs is the selection of an initial population. In our case, the initialization was done through the random selection of the values of consecutive genes.

The adaptation of the chromosomes in the population is evaluated on the basis of the adaptation function values of all the individuals in the current generation. This process takes place in several stages. First, using an appropriate decoding method, the chromosomes are converted into values of variables. Then the variables are used to calculate the objective function for all the individuals in a given population.

The stop condition is defined on the basis of the optimization requirements. In the simplest case, the performance of a set number of iterations, the testing of a proper number of points of space, or time out can be the 


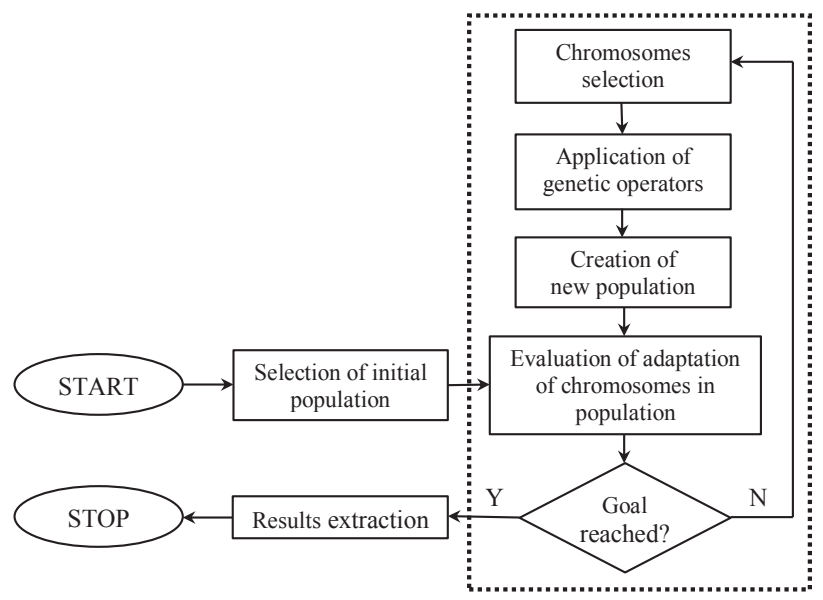

Figure 2: Genetic Algorithm operation diagram.

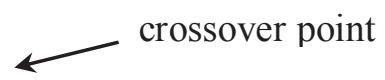

$\begin{array}{lc}\text { Parent 1 } & 001001 \mid 11011 \\ \text { Parent 2 } & 011000 \mid 10010 \\ & \downarrow \\ \text { Child 1 } & 001001 \mid 10010 \\ \text { Child 2 } & 011000 \mid 11011 \\ & \downarrow \text { crossover applied } \\ \text { Child 1 } & 001001 \mid 10000 \\ \text { Child 2 } & 011000 \mid 11011\end{array}$

Figure 3: Genetic operators.

condition. In our case, the algorithm was stopped when there was no significant improvement in the results.

The selection of chromosomes is conducted in order to isolate chromosomes for a parental pool, from which a new population (a set of points) is created using genetic operators. We used the roulette method with the scaling function. Regardless of the method used, selection is always made solely on the basis of the adaptation function values for the individuals.

The chromosomes of the individuals selected for a parental pool are subjected to the operation of genetic operators, as a result of which a population of descendants is obtained (Figure 3). We used two classic genetic operators, i.e. the crossover operator and the mutation operator. The crossover operator operates simultaneously on two chromosomes selected from a parental pool, causing the exchange of fragments of their chromosomes. Mutation is performed on one gene, with an assigned probability, causing a change in its state.

As a result of the operation of the genetic operators, a new population is formed from the individuals. This population becomes a current population and objective function and adaptation function values are calculated for it. Then the stop condition is checked and if the computations need to be continued, another cycle is started. When the stop condition is reached, the result is given as the output. The result is a set of values of the decision variables, calculated for the chromosome with the highest (when a maximum is sought) value of the objective function.

\section{Experiment}

\subsection{Soil sample}

Turoszow clays, obtained from the mine dump soil KWB Turow, due to its structural complexity, were used in the presented research. Because of its structure, this material is extremely interesting for research, and problematic from the perspective of engineering practice. The presence of water associated with the boundary surface of clay particles causes that the mechanical processes occurring in these soils are much more complex and should take into account the influence of clay particles' contact through bound water. Therefore, defining individual parameters with the greatest accuracy is a key task in the context of the modeling of engineering processes of this soil.

Basic soil parameters are:

- liquid limit: $w_{L}=37.4 \%$,

- plasticity limit: $w_{p}=26.40 \%$,

- density: $\rho=1785 \mathrm{~kg} / \mathrm{m}^{3}$,

- density of solid particles $\rho_{s}=2584 \mathrm{~kg} / \mathrm{m}^{3}$,

- initial sample soil moisture: $w=36.53 \%$,

- porosity $n=0.486$.

\subsection{Consolidation test of the sample}

The consolidation chamber used in the studies allowed the application of vertical load $P$ and measurement of displacement, filtration flow through the sample and the pore pressure in the middle of height of the sample. The scheme of the measurement station and the picture of consolidation chamber are shown in Figure 4. The load applied to the sample placed in consolidation chamber (1) 

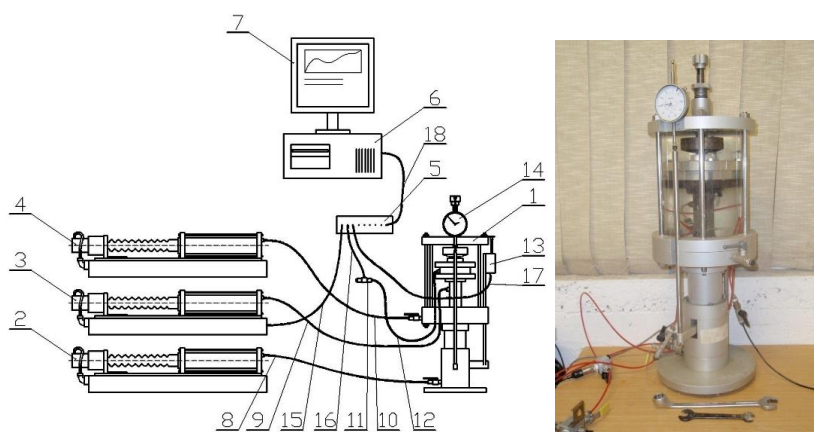

Figure 4: Components of the measuring station and connection of devices (description in text).

was produced by the regulator (2) and transmitted through a flexible tube (8). Similarly, pressures applied to the bottom and top plates are generated with the controllers (3) and (4) and transferred to the chamber through the tubes (9) and (10) respectively. The measurement of pore pressure inside the sample was measured with a pressure sensor (11) connected via a tube (12) with the interior chamber. The measurement of the progress of the consolidation was made by the displacement sensor (13) and also, for inspection, by the clock (14). Signals from the measuring devices were transferred by conductors (15), (16) and (17) to the transducer (5) where from the conditioned signal was routed with the cable (18) to PC (6). The computer allowed simultaneous automatic recording of measurement data and visualization of the experiment progress.

The presented consolidation chamber was used for the oedometer test. The pressures applied were: $12.5,25$, 50 and $100 \mathrm{kPa}$. Initial sample height $h_{0}=20 \mathrm{~mm}$ and diameter $d=75 \mathrm{~mm}$ was used. The results of the experiment are presented in Figure 5.

$$
\begin{gathered}
C_{V}=\frac{k M}{\gamma_{w}} \\
C_{V}=0.196 \frac{h^{2}}{t_{50}} \\
C_{V}=0.848 \frac{h^{2}}{t_{90}}
\end{gathered}
$$

Based on the results, the bulk modulus was calculated: $M$ $=1662$, 9KPa. Based on consolidation coefficient equation (Eq. 3), using Cassagrande (Eq. 4, Fig. 5) and Taylor (Eq. 6, Fig. 7) methods, hydraulic conductivities were calculated: $t_{50}=193,5 \mathrm{~min} ; t_{90}=524,6 \mathrm{~min}$. The calculated $k$ values are $1.189 \times 10^{-8} \mathrm{~m} / \mathrm{s}$ and $1.1898 \times 10^{-8} \mathrm{~m} / \mathrm{s}$ for Cassagrande and Taylor methods respectively.

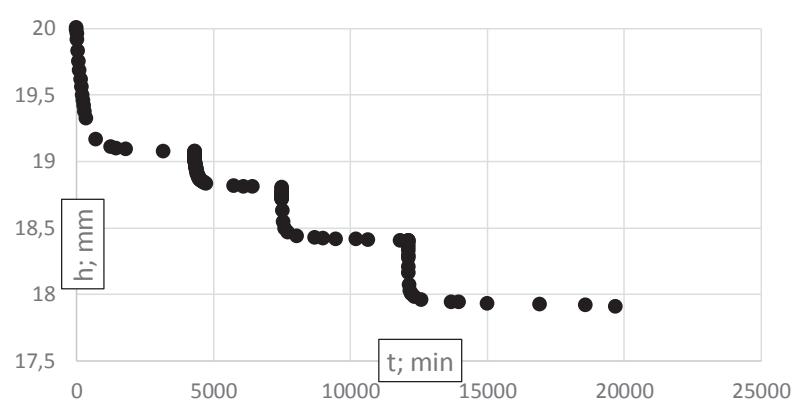

Figure 5: Oedometer test results.

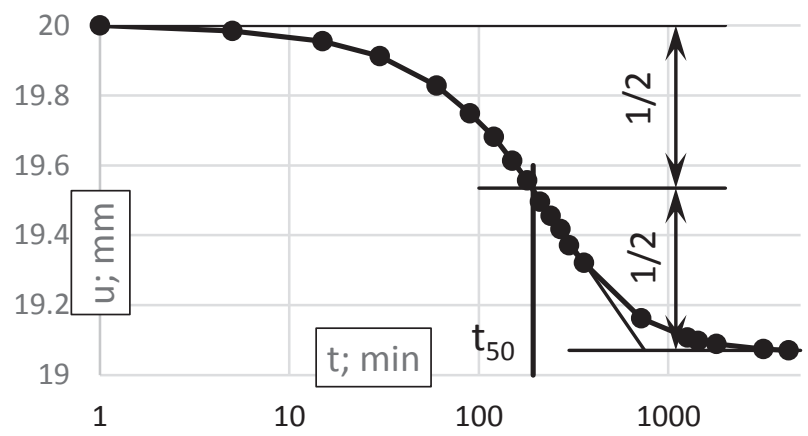

Figure 6: Cassagrande estimation of $\mathrm{Cv}$.

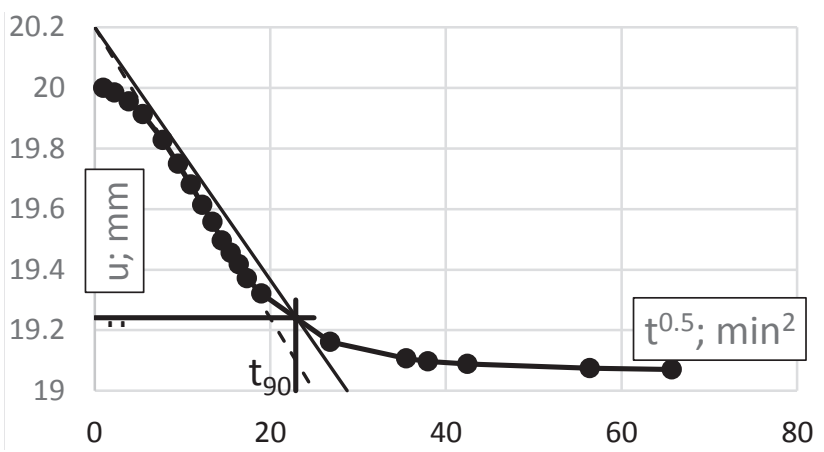

Figure 7: Taylor estimation of Cv.

\section{4 calibration}

\subsection{Methodology}

Oedometer test results were used as calibration data in the investigations. The calibration itself was carried out using a program implementing the classical genetic algorithm, designed by the authors, and the FlexPDE software for running FEM simulation needed for an inverse analysis. Thanks to this software combination, calibration can be performed exactly on the model, which is later used to 
solve actual engineering problems. In addition, owing to the FlexPDE software capabilities a complex experiment design involving the gradual loading of an oedometer sample can be used. A mathematical model was implemented in the FlexPDE program.

The algorithm was implemented in the form of an independent program written in the $\mathrm{C \#}$ language in the .NET environment. The program was written to make it possible to calibrate any mathematical model with the help of an external program supplying simulation results.

The scheme of C\# software operation in connection with the FlexPDE program is presented in Fig. 8. At the beginning of its operation, the GA reads laboratory measurement results from a file. During each cycle, GA generates parameter values and inserts it to basic pde file. Then it starts FlexPDE and instructs it to run simulation from this file and save its results. Next, GA reads simulation results and compares it with the data read earlier. The comparison is based on least square error estimator (objective function). The operation is stopped when completion criteria is met.

\subsection{Calibration results}

The calibration was performed with the use of a specially developed software implementing genetic algorithm and FlexPDE. The experimental data from four-stage oedometer test (as described in chapter 6) were used for the calibration. The mathematical model was a third Biot model with a rheological Kelvin-Voight skeleton. The subject of calibration was only one parameter $k$. To check the reproducibility of the method, 20 independent estimations were performed.

The results of the calibration are presented in Table 1. The values of hydraulic conductivities obtained varies, but the relatively small standard deviation $\sigma_{k}=4.38$ compared to average value $k_{a v}=64.75$ proves good reproducibility. The values obtained with inverse optimization technique are one order smaller than values obtained with Terzaghi model.

\section{Conclusions}

In the present paper, model calibration was carried out employing an inverse analysis using the still novel method of genetic algorithms for determining the hydraulic conductivity $k$ of the Biot-Darcy model with the rheological characteristics represented by the KelvinVoigt skeleton. The results of the investigations have proved that genetic algorithms are capable of estimating
Table 1: Parameter estimation results.

\begin{tabular}{llll}
\hline Exp. no & $\boldsymbol{k}\left[\mathbf{1 0}^{-11} \mathrm{~m} / \mathrm{s}\right]$ & Exp. $\mathbf{n o}$ & $\boldsymbol{k}\left[\mathbf{1 0}^{-11} \mathrm{~m} / \mathrm{s}\right]$ \\
\hline 1 & 70.52 & 11 & 62.43 \\
2 & 63.06 & 12 & 75.83 \\
3 & 61.44 & 13 & 61.23 \\
4 & 66.63 & 14 & 61.12 \\
5 & 62.43 & 15 & 65.01 \\
6 & 71.05 & 16 & 60.39 \\
7 & 60.70 & 17 & 61.85 \\
8 & 65.01 & 18 & 62.43 \\
9 & 66.58 & 19 & 62.43 \\
10 & 61.85 & 20 & 72.94 \\
\hline
\end{tabular}

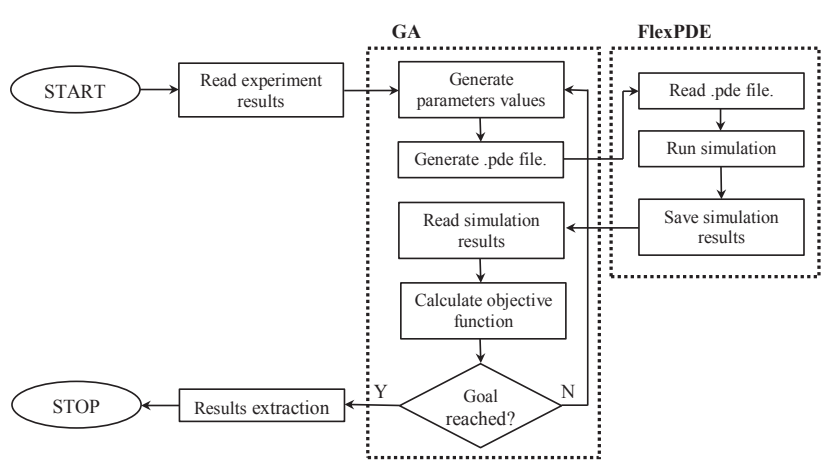

Figure 8: Scheme of operation of GA with FlexPDE.

$k$ closer to the experiment. The genetic algorithms also allow automatic model calibration and reproducibility of results, thus proving its reliability.

It has been demonstrated that genetic algorithms are a highly effective tool enabling automatic calibration based on simple rules. Experiments with genetic algorithms prove its utility for model parameter estimation. When least-squared estimator of simulation quality is used, values of hydraulic conductivity $k$ are of at least one order smaller than values obtained with the Terzaghi model. This is in the agreement with common observations that this method often leads to overestimations of $k$.

The obtained results will form the basis for further research as part of which still other rheological models and sets of experimental data can be analyzed.

\section{References}

[1] Abkenar S., Stanley S., Miller C., Chase D., McElmurry S. (2015). Evaluation of genetic algorithms using discrete and continuous methods for pump optimization of water distribution systems, 
Sustainable Computing: Informatics and Systems, Volume 8 , Pages 18-23,

[2] Anderson-Cook C. (2012) Practical Genetic Algorithms, Journal of the American Statistical Association, 100:471, 1099, DOI: 10.1198/jasa.2005.s45

[3] Auriaullt J.L.: (1991) Dynamic behaviour of porous media, Transport Processes in Porous Media, Kluver Academic Publishers, 471-519.

[4] Auriault J.L, Strzelecki T., Bauer J. He.S,: (1990) Porous deformable media by a very compressible Fluid, Eur. J. Mech. a/ Solid, 9,4,1990, pp. 373-392.

[5] Auriault J.L., Sanchez Palencia E.: (1977), Etude de comportement macroscopique d'un milieu poreux sature deformable, Journal de Mecanique,16(4), 575-603.

[6] Bartlewska M., Strzelecki T.: (2006), A methodology for determining the effective parameters of the Biot body (in Polish), 6th Scientific Conference, Szklarska Poręba, 23-25 March 2006, Research Papers of the Institute of Mining, Wroctaw University of Technology, 116, Conference Series 47.

[7] Bartlewska-Urban M., Strzelecki T.: (2008), One-dimensional consolidation of the porous medium with the rheological Kelvin-Voigt skeleton, Studia Geotechnica et Mechanica. vol. 30, No. 1/2, pp. 115-122.

[8] Bartlewska M., Strzelecki T.(2008): One-dimensional consolidation of the porous medium with the Rheological Kelvin-Voight skeleton, Studia Geotechnica et Mechanica, Vol. XXX, No. 1-2. 2008

[9] Bartlewska M.: (2009) PhD thesis entitled: Determination of the effective parameters of rheological models of cohesive soils (in Polish), Wroctaw University of Technology, Faculty of Geoengineering, Mining and Geology, Wroctaw.

[10] Bartlewska M, Strzelecki T.: (2009) Equations of Biot's consolidation with Kelvin-Voight rheological frame, Studia Geotechnica et Mechanica, Vol. XXXI, No. 2, pp. 3-15.

[11] Bartlewska-Urban M., Strzelecki T., Urban R.: (2015) Determination of effective parameters of Biot model with the Kelvin-Voight rheological skeleton, Archives of Civil and Mechanical Engineering 01/2015; DOI:10.1016/j. acme.2014.12.07.

[12] Bensoussan A., Lions J.L., Papanicolau G.: (1978), Asymptotic analysis for periodic structures, North Holland Publishing Company, Amsterdam.

[13] Biot M.A.,: (1956), General Solutions of the Equations of Elasticity and Consolidation of a Porous Material, J. Appl. Mech., 23.
[14] Biot M.A.: (1941), General theory of three-dimensional consolidation, J. Appl. Phys. No 12 s. 155,

[15] Biot M.A. Willis D.G. (1957) The Elastic Coefficients of the Theory of Consolidations, J. Appl. Mech., 24

[16] Flex PDE 6, (2009), Ver. 6, www.pdesolution.com., PDE Solutions Inc.

[17] Goldberg D., Holland J., (1988) Genetic Algorithms and Machine Learning, Machine Learning, Volume 3, Issue 2-3, 95-99

[18] Goldberg D. (1989). Genetic Algorithms in Search, Optimization, and Machine Learning. Addison-Wesley.

[19] Gen M., Cheng R. (2000), Genetic Algorithms and Engineering Optimization. John Wiley \& Sons, ISBN 0-471-31531-1.

[20] Kröner E.: (1980), Effective elastic moduli of periodic and random media: a unification, Mechanics Research Communication, 7(5), 323-327.

[21] Łydżba D., Applications of the asymptotic homogenization method in soil and rock mechanics (in Polish), Habilitation thesis, Research Papers of the Institute of Geotechnics and Hydrotechnics, Wroctaw University of Technology, ISSN 00842834, No. 23, Wrocław, Wrocław University of Technology Publishing House, 2002.

[22] Pelletier G., Chapra S., Tao H., (2006), QUAL2Kw - A framework for modeling water quality in streams and rivers using a genetic algorithm for calibration, Environmental Modelling \& Software, Volume 21, Issue 3, pp 419-425

[23] Rubinstein J., Torquato S.: (1989) Flow in random porous media: mathematical formulation, variational principles and rigorous bounds, J. Fluid Mech., 206, 25-46.

[24] Rutkowska D. (1997) Intelligent Computational Systems, PL] Academic Publishing House, Warsaw.

[25] Sastry K., Goldberg D.E., Kendall G. (2014) Genetic Algorithms. In: Burke E., Kendall G. (eds) Search Methodologies. Springer, Boston, MA

[26] Strzelecki T., Uciechowska (2014) A., Thermal consolidation process of multiphase medium consisting of elastic skeleton, water, and water vapour. Acta Geophysica vol. 62, No. 5, pp. 1163-1178. 2014. ISSN 1895-6572.

[27] Srokosz P., Selected applications of genetic algorithms in geotechnics (in Polish), Wydawnictwo UWM, 2012 ISBN: 8372997608.

[28] Zhang X., Srinivasan R., Liew M. (2009) On the use of multialgorithm, genetically adaptive multi-objective method for multi-site calibration of the SWAT model, Hydrological Processes, Volume24, Issue 8, pp. 955-969. 